\title{
Jean-François Bert, L'Atelier de Marcel Mauss. Un anthropologue paradoxal
}

Paris, CNRS Éditions, 2012, 271 p.

\section{Matthieu Béra}

\section{(Q) OpenEdition}

\section{Journals}

Édition électronique

URL : http://journals.openedition.org/assr/26314

DOI : $10.4000 /$ assr.26314

ISSN : $1777-5825$

Éditeur

Éditions de l'EHESS

Édition imprimée

Date de publication : 31 décembre 2014

Pagination : 134

ISBN : 978-2-7132-2467-6

ISSN : 0335-5985

\section{Référence électronique}

Matthieu Béra, « Jean-François Bert, L'Atelier de Marcel Mauss. Un anthropologue paradoxal », Archives de sciences sociales des religions [En ligne], 168| 2014, mis en ligne le 03 avril 2015, consulté le 22 septembre 2020. URL : http://journals.openedition.org/assr/26314 ; DOI : https://doi.org/ 10.4000 /assr.26314

Ce document a été généré automatiquement le 22 septembre 2020

(C) Archives de sciences sociales des religions 


\section{Jean-François Bert, L'Atelier de Marcel Mauss. Un anthropologue paradoxal}

Paris, CNRS Éditions, 2012, 271 p.

Matthieu Béra

\section{RÉFÉRENCE}

Jean-François Bert, L'Atelier de Marcel Mauss. Un anthropologue paradoxal, Paris, CNRS Éditions, 2012, $271 \mathrm{p}$. 
1 Assurément, on tient là un ouvrage original qui relève d'un genre auquel les sociologues ne sont pas habitués. Cet essai cherche en effet à décrire "Mauss au travail » à partir d'archives, soit exactement 52 boîtes à l'IMEC, plus sa bibliothèque conservée au Quai Branly, plus un fonds non décrit qui se trouve au Museum d'histoire naturelle. En outre, et cela étonnera les lecteurs habitués aux ouvrages d'histoire des idées, son auteur, Jean-François Bert, se préoccupe beaucoup moins des outputs de Mauss (ses publications) que de ses inputs, soit ses sources, ses «consommations intermédiaires » pour parler comme les économistes, tout ce qu'il a mobilisé en amont de l'« œuvre » - terme que récusait Mauss pour lui-même et Bert pour Mauss - en contradiction avec Victor Karady et

JEAN-RRANCOIS BERT L'ATELIER DE MARCEL
MAUSS

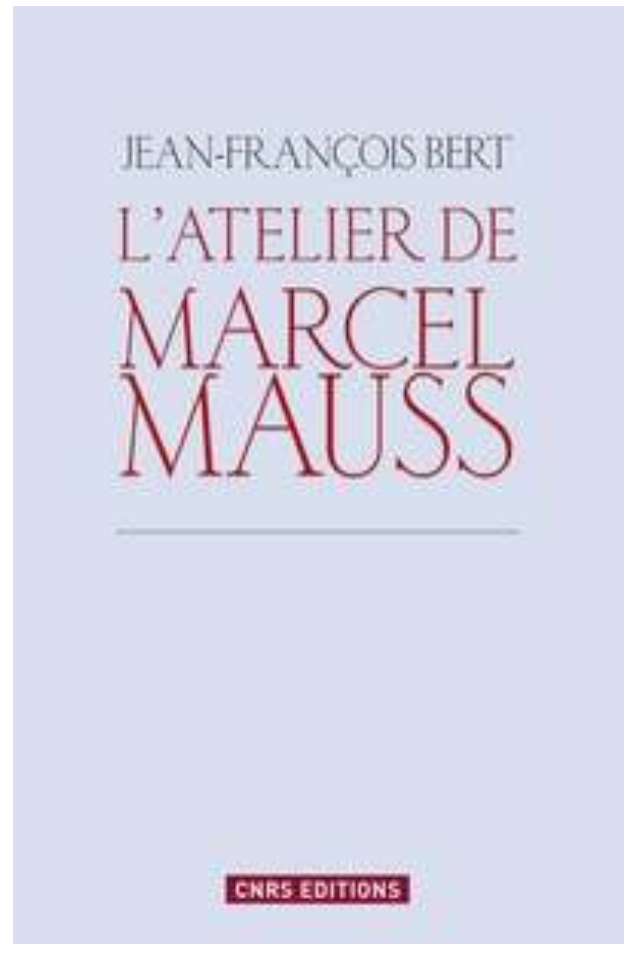
les Éditions de Minuit qui ont intitulé les trois volumes de ses textes «CEuvres» en 1969. Par principe, donc, l'essai ne revient qu'à la marge sur les écrits publiés et connus pour cela.Il nous convie dans " l'atelier » de Mauss en nous faisant partager ses «archives de chercheur ». (On pourra d'ailleurs se reporter au petit ouvrage du même, accessible en Open Edition, qui s'intitule Qu'est-ce qu'une archive de chercheur? diffusé en juin 2014). On n'apprendra rien qu'on ne sache déjà sur les théories du don, de la magie, du sacrifice, de la prière, etc. Le but n'est pas de commenter «une fois de plus" l'œuvre de «l'anthropologue paradoxal» (expression énigmatique faisant éventuellement écho avec l'article de Balandier de 1996 paru dans la Revue européenne de sciences sociales intitulé «Mauss, un itinéraire scientifique paradoxal»?). Il n'est pas non plus de se lancer dans un énième « commentaire académique ». Non. La démarche est totalement différente, innovante et par là déroutante. Elle s'inscrit dans le cadre de «l'anthropologie du travail intellectuel », un programme de recherche initié en partie par Christian Jacob à l'EHESS.

2 Pour ce faire, Bert a choisi de décomposer le «travail intellectuel» en plusieurs opérations, que reprennent les différents chapitres. Passons provisoirement par-dessus le premier d'entre eux qui vise d'autres objectifs et que nous commenterons in fine. Dans «Étudier, lire, se documenter » (chap. II), est présenté ce que les archives nous laissent à voir des trois différentes actions du travail intellectuel. Notons que d'une certaine façon, à chaque opération intellectuelle correspond un (ou plusieurs) type d'archives. Pour atteindre les traces ou les vestiges de la première opération, "Étudier ", l'approche s'intéresse aux notes de cours conservées par Mauss quand il était étudiant. Cela nous ramène donc aux années 1890-1895 pour la préparation de la licence puis de l'agrégation de philosophie, et 1895-1900 pour les études de sciences des «religions primitives». Concernant la première période, ces archives font surgir de terre des cours de Durkheim, d'Hamelin, d'Espinas qui furent ses trois enseignants de 
philosophie à Bordeaux. Mauss conserva une grande partie de ses notes toute sa vie (qui, parmi nous, pourrait se prévaloir d'en avoir fait autant?). Le travail de l'archiviste est en ce sens plus « excitant » que celui du théoricien : au plaisir de la découverte vient s'ajouter la matérialité de la preuve. Bert avait déjà édité les notes du cours de Mauss quand Durkheim enseignait Hobbes en 1894/95 (Hobbes à l'agrégation, Paris, EHESS, coll. "Audiographie ", 2011). Dans un précédent ouvrage (Penser et écrire à Deux) il avait livré quelques-unes des notes du cours de Sylvain Lévi sur le sacrifice, à l'époque où Mauss rencontra Hubert, son futur « jumeau de travail». On ne savait rien encore des cours d'Espinas, qu'on pourra utilement compléter avec l'ouvrage très précis de Jean-Claude Callède intitulé La sociologie française et la pratique sportive (1875-2005). Essai sur le sport. Forme et raison de l'échange sportif dans les sociétés modernes (Bordeaux, Maison des sciences de l'homme d'Aquitaine, 2007) qui n'est pas mobilisé ici. Il est également possible de se plonger dans les cours d'Hamelin dont les archives ont été déposées au fonds Cousin, à la Sorbonne. Les notes de cours de Mauss sont plus qu'intéressantes concernant Durkheim, dont on sait qu'on a perdu de lui tous les manuscrits. Bert évoque « des traces de plusieurs cours dont un sur la morale, un sur la certitude et un dernier sur l'association des idées»(p.60). Le fonds exhumé a aussi permis de récupérer les vieilles copies des devoirs rendus par l'étudiant Mauss, corrigées par Durkheim. On y trouve ainsi d'une main les réflexions de l'étudiant et de l'autre les appréciations de l'«oncle-maitre" sur divers sujets de philosophie. On en apprend davantage sur les relations pédagogiques entre Durkheim et son neveu, sur lesquelles on avait déjà des indications précieuses à partir de leur correspondance (Lettres à Mauss, 1998, éd. Besnard et Fournier), que Bert reprend par endroits, en les complétant par celles (inédites) adressées à Lévi ou Hubert (p. 67). De quoi reconstituer la formation de Mauss, avec évidemment des trous qui restent à combler. Par exemple, l'année 1893/1894 passée à Paris, au cours de laquelle il fit la connaissance de Fauconnet, "étudiant libre " (c'est-à-dire non boursier) comme lui et se constitua un réseau de camarades qui devinrent, pour certains, des collaborateurs de L'Année sociologique à partir de 1896/97. Quels professeurs avaient-ils, quels cours suivaient-ils alors ? Quoi qu'il en soit, on va déjà au-delà du Mauss de Fournier sorti il y a 20 ans. Les archives font avancer la science.

Dans cet atelier, "Lire» est le second volet de l'activité du chercheur. Comment retrouver, là encore, les traces d'une activité intellectuelle immatérielle? Bert nous propose pour commencer les bulletins de prêt de la Bibliothèque de Lettres de Bordeaux (p. 77) qu'il a retrouvés un peu par hasard dans les archives: ils étaient devenus des feuilles de brouillons. On a pu faire un constat identique en consultant les papiers d'Hamelin: le professeur écrivait ses cours au verso de toutes sortes d'imprimés, comme si ces universitaires manquaient de papier ! Il a ainsi pu retrouver quelques fiches d'emprunts, qu'il juge «primordiales». Il s'étonne de "l'éclectisme d'un étudiant en philosophie qui lit à quelques jours d'intervalle Guyot sur Charles $1^{\mathrm{er}}$, Brentano sur la psychologie empirique et Zeller (en allemand) sur l'histoire de la philosophie grecque. L'analyse des registres de prêts de la BU de Bordeaux » (cff. Béra et Sembel, "Les emprunts de Durkheim et Mauss à la Bibliothèque universitaire de Bordeaux, 1887-1902 ", colloque sur «la bibliothèque des savants ", au Musée du Quai Branly, février 2013, organisé par J.-F. Bert) nous montre que Mauss n'était pas si " éclectique » que cela. Il s'avère que les quelques bulletins d'emprunts conservés et retrouvés par l'auteur ne sont pas représentatifs et surtout, pris isolément, ne peuvent être caractérisés, c'est-à-dire comparés (sans perdre de vue non plus que les emprunts 
sont eux-mêmes des traces du travail de lecture, celle-ci s'effectuant sans doute pour une part bien plus considérable en salle). Notre connaissance du registre de prêts nous apprend que, sur les presque 100 emprunts de Mauss en quatre ans, on ne trouve que 21 auteurs différents. Les plus empruntés sont des auteurs de manuels (allemands): Zeller, Geschichte der deutschen philosophie zeit Leibnitz (10 fois), Joël, Beitrage zur gechichte der philosophie (5 fois) et Fisher Gechichte der neuern philosophie (13 fois). Il « faisait du manuel » comme disaient les professeurs de cette Faculté à l'époque, en le déplorant. Un seul est emprunté de manière diversifiée: Spinoza, celui-là même que Mauss envisageait d'approfondir pour son projet doctoral. On est donc loin de "l'éclectisme " pressenti par l'auteur. À côté de cela, chose assez extraordinaire, Bert nous invite à découvrir la bibliothèque privée de Mauss qui contient 21000 ouvrages, chiffre impressionnant qu'on a du mal à croire, surtout quand on veut bien le comparer avec le fonds de la bibliothèque universitaire de Bordeaux, estimé à 30000 exemplaires quand Durkheim y fut nommé en 1887 ! [Cf. Béra, "What did Durkheim borrow while in Bordeaux? » Durkheimian studies, à paraître, et aussi Durkheim à Bordeaux (1887-1902), éd. Confluences, 2014]. Cette "archive» a été conservée en grande partie. Le récit trop succinct de sa pérégrination (p. 83) nous apprend que c'est un vrai miracle que tout cela ait été sauvé en dépit de la Seconde Guerre mondiale, étant donné le "statut » de Juif pourchassé de son propriétaire, qui dut démissionner du Collège de France, porter l'étoile jaune et qui passa à travers les rafles pour des raisons que l'on ignore toujours. (On a pu évoquer la protection d'anciens étudiants qui étaient devenus des collaborateurs, c'est un mystère que la biographie de Fournier ne résout pas). Sa bibliothèque a été sauvée parce qu'elle a été déménagée chez la fille de Célestin Bouglé juste avant les confiscations, brimades et déportations. Elle a ensuite atterri au Musée du quai Branly et a été partagée avec le Musée national (p. 84). Au-delà de l'aventure d'une bibliothèque privée aussi imposante, de son inventaire qui reste à faire, que peut le chercheur qui se trouve confronté à ce gigantesque matériau ? L'auteur a commencé par étudier les annotations de Mauss, ses commentaires, les «marginalia». Des exemples forcément anecdotiques sont présentés, photographies à l'appui, comme celui qu'on trouve dans un ouvrage de Max Muller, l'un des maîtres en étude de sciences des religions. L'étude recoupe quelques-unes des annotations rageuses griffonnées en marge avec la correspondance Hubert/Mauss (p. 94-95). Ce dernier disait que Müller le " dégoutait ». Bert aurait pu se référer symétriquement aux comptes rendus de Müller qui évoquait "les deux Juifs de L'Année sociologique », ce qui aurait sans doute donné une clé explicative à l'allergie éprouvée par Mauss à l'égard de ce savant antisémite. L'acte de lecture renvoie aussi à des lieux: où Mauss lisait-il ? Comme beaucoup d'intellectuels, il passait du temps chez lui dans son « cabinet de travail » auquel (selon la correspondance avec Hubert) il apportait un grand soin. Les habitudes de travail sont importantes à connaître quand on consacre une grande partie de sa vie attablé à son bureau. Cependant, "Lire ", c'est aussi une méthode, du moins dans le cas de Mauss, qui composait des «boîtes à fiches » - il fut ainsi raillé par Charles Péguy - en vue d'organiser méthodiquement la mémoire des faits et théories croisés dans les centaines de livres consultés. On apprend dans le précédent ouvrage de Bert (Penser et écrire à deux, 2012, BB-ASSR-160), que le fichier de Hubert est toujours conservé. Il ne demande qu'à être analysé.

4 Dans le chapitre suivant (III), «Écrire et co-écrire », Bert nous conduit vers d'autres opérations intellectuelles, d'autres "gestes » et donc aussi d'autres archives. Assez bizarrement, le chapitre commence par les voyages de Mauss et se prolonge par celui 
de son ami Hubert, qu'il vécut par correspondance. En fait, Bert décide ici, sans l'énoncer clairement, de nous convier dans les correspondances de Mauss. C'était donc ce qu'il fallait entendre par « écrire ». Pourquoi ne pas avoir choisi un autre titre pour ce chapitre? «Correspondre » n'aurait-il pas mieux convenu? Sans autre explication, l'Auteur a sélectionné un extrait de la correspondance de Mauss (intégralement inédite!), avec Fauconnet, son ami de préparation à l'agrégation. S'ajoute à cela certains échanges avec Bouglé à propos de son ouvrage sur les castes. Ces morceaux choisis sont tirés de la masse de correspondance qui occupe «la moitié des 52 cartons de l'IMEC » (p. 135). La « co-écriture » avec Hubert reprend en les complétant certains éléments qu'on trouvait dans Penser et écrire à deux. Il y reparle des manuscrits, exhibe ratures et repentirs, signale les réécritures d'Hubert et de Mauss.

5 «Enseigner» (chap. IV) constitue une autre facette du travail de Mauss. Celui-ci dispensa deux séminaires par semaine à l'EPHE de 1901 à 1940, ce qui est quand même considérable, auxquels on doit ajouter les cours au Collège de France à partir de 1930, jusqu'à sa radiation en 1940 avec les lois vichystes, dont on se demande au passage ce qu'ils sont devenus. Quand on constate les efforts déployés pour publier les cours de Foucault, Barthes ou Bourdieu depuis quelques années, on reste interdit par le peu d'empressement des chercheurs à faire paraître ceux de Mauss, à dépouiller et éditer sa correspondance. Bert est parti de l'annuaire de l'École pour retrouver la liste des étudiants inscrits (p. 168). À partir de là, il a lancé son enquête pour retrouver certains d'entre eux, dans le but de récupérer leurs notes de cours. Par un fait paradoxal, en effet, autant Mauss a gardé toute sa vie les notes qu'il avait prises en assistant aux cours de ses maîtres, autant il n'a pas laissé de brouillons ou de cahiers pour ses propres cours. On irait presque jusqu'à dire que la malédiction se joue dans les deux sens : on a d'un côté les notes elliptiques d'un professeur qui ne préparait pas ses cours, du moins pas sous la forme de notes rédigées; on a perdu de l'autre les manuscrits de celui qui les rédigeait consciencieusement: Durkheim utilisait des cahiers et il évoque dans une lettre à Hubert qu'il consacrait quatre jours par semaine à élaborer les leçons d'un nouveau cours (il faut compter 22 leçons entre décembre et mai). Ici, ce ne sont donc plus les notes de cours de Mauss qu'il s'agit de relire, mais, par un joli prolongement, les notes prises par ses étudiants. On a droit à un long développement sur les notes d'André Varagnac (p. 171-184), retranscrites, sans qu'il nous soit raconté comment elles ont été retrouvées (il renvoie simplement au fonds Varagnac, MSH, Dijon, p.181, légende de la photographie), ni dans quel état général elles étaient, ni pourquoi l'auteur a sélectionné tels passages plutôt que tels autres. Il avait déjà proposé dans les Archives de sciences sociales des religions (2012, numéro sur la réception des Formes élémentaires de Durkheim) une retranscription de six autres leçons (de décembre 1922 à mai 1923, p. 179-200). Les extraits du présent ouvrage renvoient aux leçons des 22 et 28 novembre 1922 (p. 171 et 174), du 5 décembre 1921 (p. 173), des 9 et 30 janvier [1923] (p. 182-183). C'est évidemment une très belle trouvaille - cet ouvrage en est rempli, c'est ce qui lui donne tout son charme et rend haletante sa lecture - qui permet à l'auteur de faire avancer certaines questions que l'on se pose sur Mauss. Dévoiler le contenu d'un cours inédit, ce n'est ni anodin ni secondaire; cela nous dirige inévitablement vers les " outputs ", en dépit de ce qui était posé au début du livre. On n'est plus cantonné dans la seule exhumation d'archives ou dans l'interprétation pure de ce qu'est une « archive de chercheur " en soi et pour soi. On rentre dans tout autre chose, dans un travail qui va permettre de redéfinir de nombreux aspects des écrits publiés, y compris les plus connus. Cela permet aussi de découvrir la manière dont Mauss s'y prenait pour faire 
cours : il prenait un livre et le présentait à ses étudiants en leur faisant partager ses lectures. On connait ainsi les auteurs qui comptaient pour Mauss au moment de ces cours. On apprend comment il se positionnait par rapport à eux, comme en 1921 à propos de la Foi jurée de Davy, son concurrent direct sur la question du don au sein du réseau des durkheimiens ( $c f$. Besnard «Un conflit au cœur du groupe durkheimien ", Revue Française de sociologie, vol. 26, 1985). Dans ce chapitre, Bert nous fait également bénéficier d'une belle avancée à propos du fameux Manuel d'ethnographie de Mauss. Il revient sur l'édition usuelle et nous rappelle sa genèse : c'est en fait la "transcription des notes de Paulme », une étudiante de Mauss, dont on apprend qu'elle n'est pas sans poser problème (p. 186 et suiv.). Il a pu confronter ce « manuel » (c'est du moins le titre de l'édition posthume) avec les notes de Debreu qui y assista aussi (une autre année). Les différences sont importantes. Le cours d'ethnographie s'en trouve modifié. Il n'est plus le texte stabilisé qui faisait partie des classiques.

Le dernier chapitre (V) renvoie à deux autres opérations intellectuelles étrangement associées : "Chercher, éditer ». On revient alors une nouvelle fois au Mauss « lecteur », dans la mesure où "chercher» c'est en partie lire - surtout pour les "armchair ethnologists ». L'auteur s'intéresse alors aux comptes rendus que Mauss a rédigés, où s'entremêlent les actes de lire et d'écrire. Parmi les centaines d'entre eux, il met l'accent sur les recensions des ouvrages écrits par des collaborateurs de L'Année sociologique (p. 200). Il montre que Mauss s'autocensurait dans ses critiques parce qu'il les connaissait (exemples : Lapie et Hubert). On pouvait s'en douter, mais il en apporte des preuves. On repart ensuite en bibliothèque, haut lieu de la "recherche» des savants sans «terrain» (on se rend compte au passage que le plan est un peu bancal avec ses allers-retours, sans doute parce que le thème du chapitre, "chercher ", est bien trop vague et mal choisi, vu qu'il englobe à lui seul toutes les opérations à décomposer). Il se trouve que Mauss avait conservé de son séjour à la British Library où il s'était rendu en 1898, 1904 et 1921, des «liasses de fiches d'emprunts» (p. 206). L'Auteur les a classées chronologiquement et a étudié ce que Mauss lut alors (p. 204-222). Ce passage est peut-être le plus fouillé du livre grâce à son véritable travail de classement des emprunts, entre le 14 et le 24 octobre 1924, concernant 77 ouvrages (certains réitérés). On mesure ainsi la méthode et la capacité de travail de Mauss: 77 ouvrages consultés en 10 jours, cela représente onze ouvrages par jour et surtout autant qu'en quatre années d'emprunts à la BU de Bordeaux! Cette incursion approfondie en dit long sur la faible représentativité des emprunts stricto sensu si l'on cherche à atteindre par là l'activité de lecture. Les traces du travail en salle de lecture qui sont livrées ici à l'historien par ces bulletins de demande de communications d'ouvrages sont bien plus pertinentes, que ce à quoi donnent accès les registres d'emprunts des bibliothèques. Malheureusement, elles ne sont presque jamais conservées par les institutions. L'auteur propose un regroupement par zone géographique et repère que Mauss voulait découvrir les dernières parutions sur le Ghana, l'Australie ou l'histoire du judaïsme. Il signale aussi que ces lectures extensives (comme on parle de "culture extensive») ne seront pas toujours reprises dans ses travaux (p. 210). On a la preuve, s'il en fallait, que le chercheur réalise un travail invisible au sens où il n'est pas directement "productif». Bert s'arrête aussi un moment sur Sir Grafton Eliot Smith, dans la mesure où Mauss le lit avec une attention renouvelée. Il recoupe ces lectures avec un compte rendu donné à L'Année sociologique ( Cuvres, 1969, II, p. 514). Il revient sur le contenu de l'œuvre, puisqu'il compare des lectures avec des « productions publiées ». S'il a choisi de se focaliser sur Smith (p. 125), 
c'est parce que Mauss possédait aussi des ouvrages de cet auteur dans sa bibliothèque personnelle. Quand trois faisceaux se croisent, cela enrichit considérablement le travail historique. Bert n'a pas été jusqu'à lire lui-même l'auteur en question - c'eut été un quatrième faisceau - tant il est difficile de suivre l'érudition exceptionnelle de Mauss.

7 "Éditer " achève dans le même chapitre, L'atelier de Mauss. Mauss passa des années à éditer des ouvrages posthumes d'amis décédés : de Hertz tué à la guerre (5 pages), de Hubert décédé dans les années 1920 (10 pages), sans oublier son oncle Durkheim (2 pages seulement). Cela faisait partie de la posture de Mauss, de son rapport à la mémoire de ses anciens amis, de ses maîtres, de ses disciples ou ses collaborateurs. Les archives sont ici sollicitées à travers la correspondance que ce travail a pu entraîner avec les éditeurs.

8 Après avoir restitué l'essentiel de ce beau et passionnant ouvrage qui fourmille de données nouvelles sur Mauss, qu'il nous soit permis de formuler quand même deux remarques. Sur l'opposition que l'auteur manifeste à l'égard du genre biographique d'abord. Sa virulence envers les biographies " académiques » nous semble excessive et surtout maladroite, compte tenu de ce qu'il propose lui-même. Le premier chapitre que nous avions laissé de côté expose la position de l'auteur : il est sur le même thème et le même ton que l'introduction et la conclusion (p. 241-247). À Fournier, «absolument incontournable» (p.26), il adresse la même critique que Lukes lui reprochant d'avoir « laissé le lecteur à l'orée de la tâche », d'avoir mis de côté les grandes questions posées par l'œuvre de Mauss. Mais cette reprise de la critique de Lukes n'est-elle pas paradoxale quand elle vient de celui qui décide de ne s'intéresser qu'aux inputs sans chercher à réinterpréter les outputs? Ce travail apprend, mais à condition de le relier à ce qui se pense, s'écrit et se publie, circule et s'utilise (ce serait encore un autre point d'approche, comme nous le propose par exemple Marc Joly dans Devenir Elias, Fayard, 2012). Il n'est pas incompatible avec une biographie intellectuelle, ni avec des « interprétations académiques ». Les trois approches nous paraissent complémentaires, plutôt qu'exclusives les unes des autres. Une seconde remarque : l'auteur veut, dans le prolongement des travaux de l'école d'Edimbourg, étudier « la science en action » (cf. le titre de la conclusion : " Pour une histoire du travail savant en action : les archives de chercheur »). Est-ce possible d'atteindre ce but à partir d'archives ? Le propre de la méthode de Latour et Woolgar (La Vie de laboratoire, 1978) est de suivre la pratique savante en l'observant in vivo. Comment faire cela quand on ne peut ni observer ni interroger puisque tout est terminé ? Comment, à partir de simples traces, dont la représentativité même nous échappe en partie, à partir de papiers gardés selon des logiques qui tiennent à des raisons peu défrichables (sans même parler du hasard, bête noire de la science...), prétendre réaliser ce "programme fort »? L'historien peut-il être aussi un anthropologue du travail intellectuel ? N'a-t-on pas davantage à faire avec ce qui serait de la paléontologie? Que nous apprend vraiment la description des vestiges d'un atelier désaffecté depuis longtemps, démembré, éparpillé, sur la production qui en sortit? Autant de questions qui méritent d'être posées aux historiens, aux biographes, aux historiographes. 